\title{
Status and prospects of the SNO+ experiment
}

\author{
Ana Sofia Inácio for the SNO+ Collaboration ${ }^{a, b, *}$ \\ ${ }^{a}$ Laboratório de Instrumentação e Física Experimental de Partículas, \\ Av. Prof. Gama Pinto 2, 1649-003 Lisboa, Portugal \\ ${ }^{b}$ Department of Physics, University of Lisbon, \\ Edifício C8, Campo Grande 1749-016 Lisboa, Portugal \\ E-mail: ainacio@lip.pt
}

$\mathrm{SNO}+$ is a large volume liquid scintillator experiment for neutrino physics located at SNOLAB, Canada. Using the 12-m diameter acrylic vessel and the PMT array of the SNO detector, SNO+ has the primary physics goal of searching for the neutrinoless double-beta decay of the ${ }^{130} \mathrm{Te}$ isotope. Data collected will also be used to explore additional physics topics such as reactor antineutrino oscillations, solar neutrinos, geoneutrinos, and to search for exotic physics. SNO+ completed its first phase as a pure-water Cherenkov detector, which focused on detector commissioning and calibration, and published its first physics results. Currently, the detector is completely filled with liquid scintillator, to which 1.3 tonnes of ${ }^{130} \mathrm{Te}$ is expected to be loaded in late 2022 .

Particles and Nuclei International Conference - PANIC2021

5 - 10 September, 2021

Online

${ }^{*}$ Speaker 


\section{The SNO+ Experiment}

SNO+ is a multi-purpose, low background liquid scintillator experiment for neutrino physics, located $2 \mathrm{~km}$ underground at SNOLAB in Canada [1, 2]. The detector re-uses most of the components of the Sudbury Neutrino Observatory (SNO) that operated from 1999 to 2006 [3], with several major upgrades to enable the use of liquid scintillator as target material.

The detector, shown in Figure 1, consists of a spherical acrylic vessel (AV) with a thickness of $55 \mathrm{~mm}$ and a radius of $6 \mathrm{~m}$, which contains the detector target medium. The AV is surrounded by a $\sim 8 \mathrm{~m}$ radius geodesic steel structure holding 9362 inward-facing photomultiplier tubes (PMTs). The volume outside the AV, including the cavity into which the detector is inserted, is filled with 7000 tonnes of ultra-pure water, shielding against the radioactivity from instrumentation and the surrounding rock. The upgrades from SNO to SNO+ included the addition of a holddown rope system to counter the buoyancy of the $\mathrm{AV}$ when it is filled with liquid scintillator; upgraded electronics to handle the higher data taking rates; and new calibration hardware to meet the radiopurity and compatibility requirements for the scintillator.

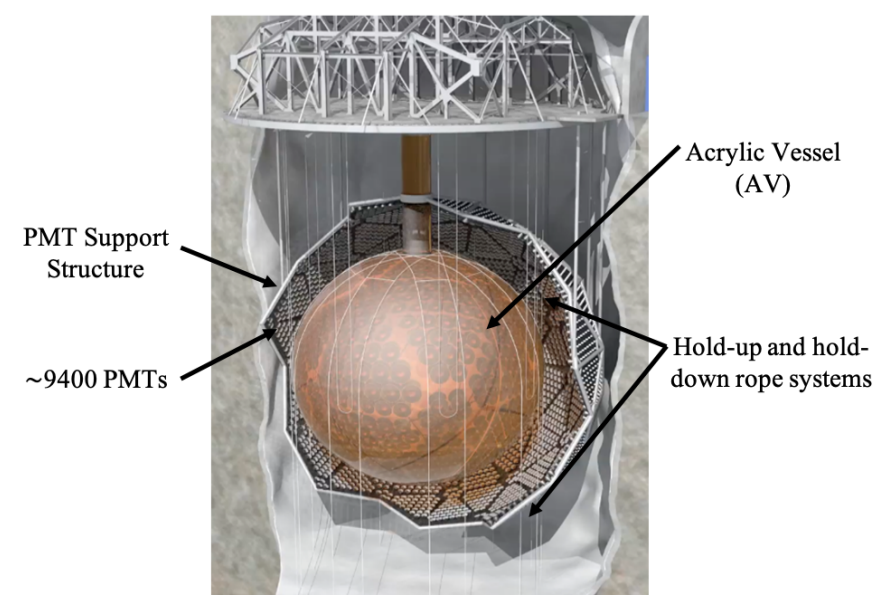

Figure 1: Schematic of the SNO+ detector.

SNO+ acquired data as a pure-water Cherenkov detector between May 2017 and June 2019. The water has now been replaced by 780 tonnes of liquid scintillator for the second phase of the experiment. After a few months of data taking in this configuration, the scintillator will be loaded with 3.9 tonnes of natural tellurium $(0.5 \%$ by weight $)$ to search for the $0 v \beta \beta$-decay of ${ }^{130} \mathrm{Te}$. Due to its large volume and high radio-purity, the $\mathrm{SNO}+$ experiment will also explore several other physics topics, such as searching for nucleon decay, measurements of geoneutrinos, reactor antineutrinos, and solar neutrinos. SNO+ also aims at monitoring along all the phases possible supernova neutrino bursts.

\section{Results from the Water Phase}

The SNO+ water phase was dedicated to measuring the external background sources, testing the data acquisition systems and detector performance. The detector was calibrated using both optical and radioactive sources, which were deployed in many positions inside and outside the AV. The 
absolute energy scale and position resolution were determined using a tagged ${ }^{16} \mathrm{~N}$ gamma source [4], yielding a $10 \mathrm{~cm}$ position resolution and a $1 \%$ uncertainty for the energy scale. A deployed light diffusing sphere (laserball) was used to measure water attenuation coefficients, effective attenuation coefficients for the AV, and the angular response of the PMTs, across different wavelengths. These measurements, published in [5], improved the detector response model, in particular its variation with position, to better than $0.6 \%$. An Am-Be calibration source was used to measure $2.2 \mathrm{MeV}$ $\gamma \mathrm{s}$ produced by neutron capture on hydrogen. These measurements, published in [6], revealed a neutron detection efficiency of 50\%, the highest efficiency achieved among pure water Cherenkov detectors to date.

The SNO+ water phase includes two main physics datasets with 115 and 190 live days. The analysis of the first dataset, collected between May and December 2017, led to two publications. The first publication [7] reported the observation of elastic scattering of ${ }^{8} \mathrm{~B}$ solar neutrinos, with very low backgrounds above $6 \mathrm{MeV}$. The measured flux was $2.53_{-0.28}^{+0.31}$ (stat. $)_{-0.10}^{+0.13}$ (syst.) $\times 10^{6} \mathrm{~cm}^{-2} \mathrm{~s}^{-1}$, consistent with measurements by other experiments. The second publication [8] involved a search for invisible nucleon decay. SNO+ was able to set the world's best livetime limits of $3.6 \times 10^{29}$ years for invisible proton decay, and of $2.6 \times 10^{28}$ years for $p n$ and $4.7 \times 10^{28}$ years for $p p$ decay. These results will be soon updated by including the completed optical calibration and additional data from the second dataset, which has much lower backgrounds.

\section{Transition to the Scintillator Phase and Current Status}

In 2019 the water inside the AV started being replaced with liquid scintillator, consisting of linear alkylbenzene (LAB) as solvent and 2,5-diphenyloxazole (PPO) as fluor. The development, characterization and deployment of the SNO+ liquid scintillator is documented in [9]. Due to the COVID-19 global pandemic, there was no access to the mine where SNOLAB is located for several months, and the scintillator fill had to be paused. SNO+ took data for 7 months (from March to October 2020) half-filled with 365 tonnes of LAB, with a PPO loading of $0.5 \mathrm{~g} / \mathrm{L}$. Filling operations restarted in October 2020, and as of March 2021 the detector is fully loaded with scintillator. Currently, a campaign to complete the loading of PPO is ongoing.

The partial fill period provided the ideal opportunity to start measuring the backgrounds in the liquid scintillator, and to prepare and test the physics analysis tools for the scintillator phase. The detector response was monitored using calibration sources deployed outside the $\mathrm{AV}$, in order to leave the scintillator undisturbed and avoid contamination. In-situ ${ }^{214} \mathrm{Bi}^{214} \mathrm{Po}$ tagged coincidences provided additional calibration points to determine the scintillator light yield, and were used to tune the $\alpha$ and $\beta$ scintillator timing profile and quenching parameters in the Monte Carlo, necessary for an accurate event reconstruction. The measured light yield at $0.5 \mathrm{~g} / \mathrm{L}$ of PPO was $\sim 300$ p.e. $/ \mathrm{MeV}$, consistent with expectations.

During the partial fill period, the radon that ingressed the detector during the fill decayed away, allowing to determine the intrinsic Uranium and Thorium levels in the scintillator. The measured concentrations were $(4.7 \pm 1.2) \times 10^{-17} \mathrm{gU} / \mathrm{g}$ and $(5.3 \pm 1.5) \times 10^{-17} \mathrm{gTh} / \mathrm{g}$, which are below the requirements for the $0 v \beta \beta$-decay searches. The partial fill data were used to start the scintillator phase physics program. There is an on-going geo and reactor anti-neutrino analysis, and a measurement of ${ }^{8} \mathrm{~B}$ solar neutrinos was performed (Figure 2), yielding a flux of 
$5.66 \times 10^{6}{ }_{-18.2 \%}^{+20.6 \%}$ (stat. $)_{-7.4 \%}^{+6.3 \%}$ (syst.) $\mathrm{cm}^{-2} \mathrm{~s}^{-1}$ with an exposure of $22.3 \mathrm{kt}$-day, compatible with other measurements $[7,10]$. The low PPO concentration scintillator data are being used to explore Cherenkov/scintillation light separation and directionality on an event-by-event basis, which would improve future solar neutrino measurements with these data.

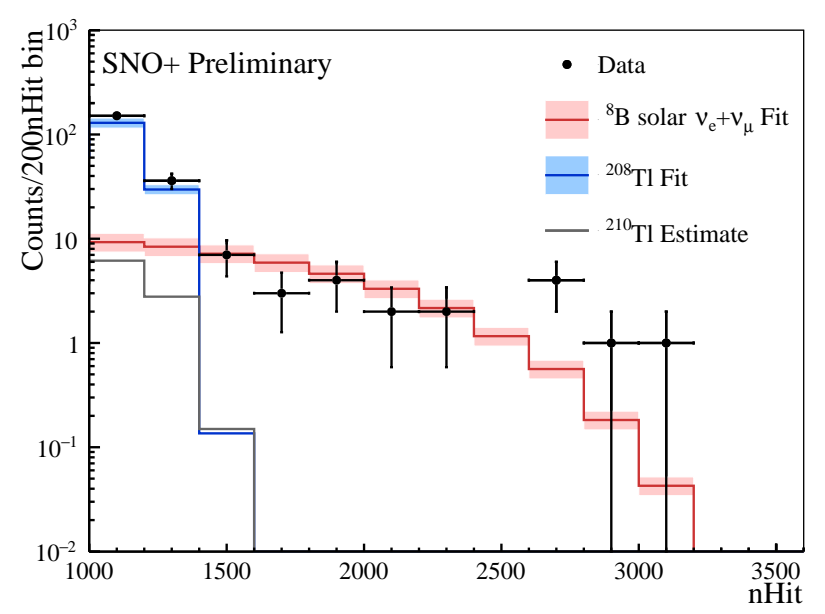

Figure 2: Fitted nHit spectrum of the partial fill data for ${ }^{8} \mathrm{~B}$ solar neutrino elastic scattering and backgrounds. nHit corresponds to the number of hit PMTs, proportional to energy ( $\sim 300 \mathrm{hits} / \mathrm{MeV})$. The red line shows the best fit signal, and the blue and grey lines the ${ }^{208} \mathrm{Tl}$ and ${ }^{210} \mathrm{Tl}$ backgrounds, respectively. The ${ }^{210} \mathrm{Tl}$ normalization was constrained in the fit. The error bands include statistical and systematic uncertainties.

\section{Prospects for the Tellurium Phase}

SNO+ developed an innovative technique for loading the natural Tellurium into the scintillator, while maintaining good optical properties and high light yield. Telluric acid, $\mathrm{Te}(\mathrm{OH})_{6}$, will be mixed with 1,2-Butanediol, forming tellurium-butanediol (TeBD) complex, which is miscible with LAB. A secondary wavelength shifter, bis-MSB, will be added to the mixture, as well as a stabilizer called Dimethyldodecylamine (DDA). The telluric acid will be purified in a purification plant underground, to further reduce cosmogenic isotopes and other impurities. It will then be mixed into TeBD and loaded into the scintillator. The purification targets for the final tellurium cocktail are $10^{-15}$ and $10^{-16} \mathrm{~g} / \mathrm{g}$ of $\mathrm{U}$ and $\mathrm{Th}$ respectively.

The chosen energy region of interest (ROI) for the $0 v \beta \beta$ is centered around the $2.5 \mathrm{MeV}$ Qvalue and is asymmetric ( $-0.5 \sigma$ to $1.5 \sigma)$ due to the presence of the irreducible $2 v \beta \beta$ background at its lower edge. About 9.5 background events are expected in the ROI per year, in a fiducial volume of 3.3-m radius. The main contributions to this number are (in order of decreasing contribution): ${ }^{8} \mathrm{~B}$ solar neutrino elastic scattering events; internal $\mathrm{U}$ and Th chain radioactivity; external gamma rays from the PMTs, support ropes, $\mathrm{AV}$, and external water; the tail of the $2 v \beta \beta$, and cosmogenic backgrounds. In an optimized energy ROI and fiducial volume, and from a simple counting analysis, the expected half-life sensitivity after 5 years of data is $2.1 \times 10^{26}$ years at $90 \%$ confidence level, corresponding to an effective Majorana mass, $m_{\beta \beta}$, in the range from 37 to $89 \mathrm{meV}$. One of the main advantages of the $\mathrm{SNO}+$ technique is the possibility of moving toward higher sensitivities 
by increasing the Tellurium loading. R\&D efforts have demonstrated that the loading could be increased up to $3 \%$ (by weight) while maintaining a good light yield and stability.

\section{Summary}

The SNO+ experiment successfully completed its water commissioning phase and, since March 2021, the detector is completely filled with unloaded liquid scintillator. The tellurium loading is scheduled to start in late 2022.

\section{Acknowledgments}

This work is funded by EGI, GridPP, Compute Canada, CFI, STFC, NSERC, CFI, CIFAR, FCT, Deutsche Forschungsgemeinschaft, ERC, DOE, NSF, Berkeley, ASRIP, Ontario MRI, FedNor, Queen's University. The presenter Ana Sofia Inácio is funded by FCT (Fundação para a Ciência e Tecnologia), Portugal, through the research grant PTDC/FIS-NUC/0640/2014 and through the IDPASC PhD grant PD/BD/135468/2017. We thank SNOLAB and Vale for their valuable support.

\section{References}

[1] S. Andringa et al. (SNO+ Collaboration), Current Status and Future Prospects of the SNO+ Experiment, Adv. High Energy Phys. 2016 (2016) 6194250, [arXiv:1508.05759].

[2] V. Albanese et al. (SNO+ Collaboration), The SNO+Experiment, JINST 16 (2021) P08059 [arXiv:2104.11687].

[3] J. Boger et al. (SNO Collaboration), The Sudbury Neutrino Observatory, Nucl. Instr. and Meth. A 449 (2000) 172-207, [arXiv:nucl-ex/9910016].

[4] M. R. Dragowsky et al. (SNO Collaboration), The ${ }^{16} N$ calibration source for the Sudbury Neutrino Observatory, Nucl. Instrum. Meth. A 481 (2002) 284-296, [ arXiv:nucl-ex/0109011].

[5] M. R. Anderson et al. (SNO+ Collaboration), Optical calibration of the SNO+ detector in the water phase with deployed sources, JINST 16 (2021) P10021, [arXiv:2106.03951].

[6] M. R. Anderson et al. (SNO+ Collaboration), Measurement of neutron-proton capture in the SNO+ water phase, Phys. Rev. C 102 (2020) 014002, [arXiv:2002.10351].

[7] M. R. Anderson et al. (SNO+ Collaboration), Measurement of the ${ }^{8} B$ solar neutrino flux in SNO+ with very low backgrounds, Phys. Rev. D 99 (2019) 012012, [arXiv:1812.03355].

[8] M. R. Anderson et al. (SNO+ Collaboration), Search for invisible modes of nucleon decay in water with the SNO+ detector, Phys. Rev. D 99 (2019) 032008, [arXiv:1812.05552].

[9] M. R. Anderson et al. (SNO+Collaboration), Development, characterisation, and deployment of the SNO+ liquid scintillator, JINST 16 (2021) P05009, [arXiv:2011.12924].

[10] B. Aharmim et al. (SNO Collaboration), Combined Analysis of all Three Phases of Solar Neutrino Data from the Sudbury Neutrino Observatory, Phys. Rev. C 88 (2013) 025501 , [arXiv:1109.0763]. 